\title{
Effects of Pulsed Electric Field Processing on Microbial Survival, Quality Change and Nutritional Characteristics of Blueberries
}

Tony Z. Jin ${ }^{\mathrm{a}}$, Yuanshan Yu ${ }^{\mathrm{b}}$, Joshua B. Gurtler ${ }^{\mathrm{a}}$

${ }^{a}$ U.S. Department of Agriculture, Agricultural Research Service, Eastern Regional Research Center, 600 East Mermaid Lane, Wyndmoor, PA 19038, USA

${ }^{\mathrm{b}}$ Sericultural \& Agri-Food Research Institute, Guangdong Academy of Agricultural Sciences, No.133, Yihenglu, Guangzhou 510610, China.

* Corresponding author: Dr. Tony Jin, e-mail: tony.jin@ars.usda.gov.

₹ Mention of trade names or commercial products in this article is solely for the purpose of providing specific information and does not imply recommendation or endorsement by the U.S. Department of Agriculture. USDA is an equal opportunity provider and employer. 


\begin{abstract}
Whole fresh blueberries were treated using a parallel pulsed electric field (PEF) treatment chamber and a sanitizer solution (60 ppm peracetic acid [PAA]) as PEF treatment medium with square wave bipolar pulses at $2 \mathrm{kV} / \mathrm{cm}$ electric field strength, $1 \mu$ s pulse width, and 100 pulses per second for 2, 4, and 6 min. The effects of PEF on native microbiota and artificiallyinoculated Escherichia coli K12 and Listeria innocua populations on blueberries were determined. Color, texture, anthocyanins and total phenolic compound concentrations were also evaluated. The combination of PEF and PAA was able to achieve up to 3 log reduction of $E$. coli and Listeria as well as $2 \mathrm{log} / \mathrm{g}$ reduction of native microbiota. PEF treatments did not cause any changes in color and appearance of the blueberries. The treatments did, however, cause the blueberries to soften in texture. Anthocyanins and phenolic compounds in blueberries increased by 10 and $25 \%$, respectively, after PEF treatments. The results demonstrate the potential of PEF applications to enhance the safety and improve the quality and nutritional value of fruits and their derived products.
\end{abstract}

Key words: pulsed electric fields, blueberries, microbial, quality, nutrition, sanitizer

Chemical compounds studied in this article Peroxyacetic acid (PubChem CID: 195769); Hydrogen peroxide (PubChem CID: 784); Sodium chloride (PubChem CID: 5234) 


\section{Introduction}

Small fruits such as raspberries and blueberries are high value commodities for their well-reported health benefits. In terms of U.S. fruit consumption, blueberries rank only second to strawberries in popularity of berries. Blueberries are not only popular, but also repeatedly ranked in the U.S. diet as having one of the highest antioxidant capacities among all fruits, vegetables, spices and seasonings. Antioxidants are essential to optimizing health by helping to combat the free radicals that can damage cellular structures as well as DNA (Whfoods, 2016).

Berry fruits are usually grown in open fields and are constantly exposed to sources of potential pre-harvest contamination such as soils, insects, birds, and irrigation water as well as human contact during harvesting. Therefore, they are susceptible to contamination caused by pathogenic bacteria. In recent years, an increasing number of foodborne illness outbreaks has been associated with small fruits such as raspberries and blueberries (Calder et al., 2003; Luna \& Griffin, 2010; Miller et al., 2013; Sarvikivi et al., 2012). Two outbreaks and 52 illnesses were related to the consumption of blueberries in 2014 (CDC, 2015). In addition, the warm temperature during the harvest season prompts fruit spoilage. Wu and Kim (2007) observed that visible signs of decay can develop on warm, wet blueberries in less than $12 \mathrm{~h}$. Therefore, proper intervention controls are needed and should be applied as soon as the fruits are harvested. The intervention processes currently being used may not be sufficient for controlling the biological hazards in these foods, creating urgency for the development of new intervention technologies.

Fruit washing with sanitizers has been used by farms, processors, and distributors to reduce initial microbial loads, but the effectiveness has been limited. Chlorinated water sprays (100 ppm) have demonstrated reductions of less than $1 \log$ CFU/g against APC on the surface of low bush blueberries, when compared to unwashed berries (Crowe et al., 2005). Alexandre et al. 
(2012) reduced total aerobic mesophilic bacteria on strawberries by 1.5 and $2.2 \log$ CFU with $1 \%$ and 5\% hydrogen peroxide, respectively. Hung et al. (2010) reduced E. coli O157:H7 populations artificially inoculated onto strawberries by $1.17 \log \mathrm{CFU} / \mathrm{g}$ when treated with electrolyzed oxidizing water at $4^{\circ} \mathrm{C}$ for 1 minute, and by $1.58 \log \mathrm{CFU} / \mathrm{g}$ when the treatment time was increased to 5 minutes. When ultrasonication was added, E. coli $\mathrm{O} 157: \mathrm{H} 7$ populations on strawberries were further reduced by 1.49 and $1.89 \log$ CFU/g, respectively. The efficacy of electrostatic sprays of electrolyzed oxidizing water, UV light, ozone, and a combination of ozone and UV light in inactivating E. coli O157:H7 artificially inoculated onto blueberries was studied and a synergistic effect for the combination was observed (Kim \& Hung, 2012). Tsunami® 100 is an Environmental Protection Agency (EPA) registered antimicrobial water additive for pathogen reduction in fruit and vegetable processing water, composed of acetic acid, peracetic acid and hydrogen peroxide. Tsunami 100 is recommended for use in the process waters of post harvest, fresh cut, and processed fruits and vegetables in both batch and continuous operations. The Environmental Protection Agency (EPA (1998) authorizes the use of peroxyacetic acidbased additives for fruits and vegetables and washed water up to $80 \mathrm{ppm}$. Alvaro et al. (2009) used the peracetic acid sanitizer (Tsunami ${ }^{\circledR} 100$, Ecolab) for sweet pepper, tomato and cucumber (500 $\mathrm{mg} / \mathrm{L}$ for $2 \mathrm{~min}$ ) and stated that the perorganic acid compound is better for washing fruit and improving postharvest shelf life as it is environmentally friendly, breaking down into acetic acid and oxygen after use, does not have any adverse effects on human health, and does not affect the taste characteristics of fruits and vegetables.

PEF treatments have been studied for killing foodborne pathogenic microorganisms, such as E. coli O157:H7, Salmonella spp. and L. monocytogenes as well as spoilage bacteria, molds and yeasts (Guo et al., 2014; Gurtler et al., 2011, 2010; Jin et al., 2015, 2014, 2009a, 2009b, Min 
et al., 2003; Reina et al., 1998). PEF treatments have exhibited the potential of maintaining the physico-chemical quality of liquid food products, without substantially impacting the sensory properties and health-related compound makeup (Guo et al., 2014; Jin \& Zhang, 1999). Most of these studies have been focused on liquid foods, such juices and beverages. There is an increasing interest in PEF pretreatment of fresh produce products, such as potato and apple (Angersbach et al., 2000; Bazhal et al., 2001; Lebovka et al., 2004). However, there is no information available for inactivation of microbial contaminants on fresh whole fruits, particularly combined with the use of a sanitizing solution as a PEF treatment medium, and their impact on microbial reduction, product quality and nutritional value of whole berry fruits. Fresh berries are, traditionally, a seasonal food, and their consumption is highest during the summer months. Frozen berries, berry jams, juices/nectars, and dehydrated berries are all typically available at other times of the year.

The central idea behind this current study was to evaluate PEF processing for its potential use as a pretreatment that would extend the shelf-life, enhance the safety and improve nutritional and other characteristics of whole fresh fruits and their derived constituent products (i.e., juice, puree, jam, pastes, raisin and other dehydrated products). The specific objectives of this first study were to: 1) investigate the antimicrobial efficacy of PEF treatment in combination with sanitizing solution for blueberries; 2) evaluate the effects of these treatments on the quality and nutritional attributes of fresh fruits, and 3) assess the potential of these treatments to manufacture derived products.

\section{Materials and Methods}

\subsection{Blueberry preparation}


Fresh blueberries were obtained from local supermarkets, stored in a refrigerator at $4{ }^{\circ} \mathrm{C}$ until the time of the experiment and used within $c a$. 1 week of purchasing. Blueberries with a diameter of about 12-14 $\mathrm{mm}$ and average weight of $2 \mathrm{~g} /$ piece were selected for the experiments.

\subsection{Inoculation of blueberries}

E. coli K12 and Listeria innocua were used as surrogates of pathogenic E. coli $\mathrm{O} 157: \mathrm{H} 7$ and Listeria monocytogenes, respectively. E. coli K12 (ATCC 23716) and Listeria innocua (ATCC 33090) were obtained from the culture collection of the U.S. Department of Agriculture, Agricultural Research Service, Eastern Regional Research Center (Wyndmoor, PA, USA). Frozen stock cultures of each strain were cultured independently in $9 \mathrm{~mL}$ Tryptic Soy Broth (TSB, BBL/Difco Laboratories, Sparks, MD, USA) in sterile $150 \mathrm{~mm}$ glass tubes at $37^{\circ} \mathrm{C}$ for $18 \mathrm{~h}$.

For studies involving the evaluation of quality and nutritional properties or natural microbiota, blueberries were only washed with tap water. For studies involving the artificial inoculation of Listeria or E. coli, blueberries were further sprayed with $70 \%$ ethanol to remove background microorganisms and dried for $2 \mathrm{~h}$ in biohood prior to inoculation. Blueberries were immersed into $100 \mathrm{~mL}$ of the inoculum for $1 \mathrm{~min}$ and then placed on a sterile tray in a laminar flow hood and dried under continuous circulating laminar flow for $2 \mathrm{~h}$ at $22 \pm 2{ }^{\circ} \mathrm{C}$ to facilitate microbial attachment on the fruit surface.

\subsection{Sanitizing solution and PEF treatment}

Tsunami 100 (EcoLab, St. Paul, MN) (15.2\% peroxyacetic acid, 11.2\% hydrogen peroxide) was used in this study. One liter of sterile distilled water was conditioned at $22^{\circ} \mathrm{C}$ 
overnight, and $250 \mu 1$ or $500 \mu 1$ Tsunami 100 (T100) was added to the sterile distilled water just before each experiment. The concentration of peroxyacetic acid in each sanitizing solution was determined using a test kit acceding to the manufacturer's instruction. The concentration of peroxyacetic acid corresponding to $0.25 \%$ and $0.5 \%$ solutions were 30 and $60 \mathrm{ppm}$, respectively.

A bench-scale PEF processing system (OSU-4H Model) located at the Eastern Regional Research Center, Agricultural Research Service, USDA (Wyndmoor, PA, USA) was used for this study (Figure 1). The system provided bipolar square waveform pulses with a maximum peak voltage of $\pm 11 \mathrm{kV}$. The high voltage pulse generator operated at a maximum repetition rate of 2000 pulses per second and a pulse width of $1 \sim 10 \mu$ s. Pulses were monitored with a high voltage probe (VD-60; Northstar, Albuquerque, NM, USA), current monitors (Model 110; Pearson, Palo Alto, CA, USA) and oscilloscopes (TDS-210; Tektronix, Beaverton, OR, USA). A specially designed parallel-electrode (stainless steel) PEF treatment chamber with $2.5 \mathrm{~cm}$ gap distance and an area of $56 \mathrm{~cm}^{2}(7 \times 8 \mathrm{~cm})$ was used to treat blueberry samples (Figure 1).

Before PEF treatment, fresh blueberries (30 pieces, ca. $60 \mathrm{~g}$ ) were placed in the treatment chamber, and then the chamber was filled with the salt solution $(0.025 \% \mathrm{w} / \mathrm{v} ; 500 \mu \mathrm{S} / \mathrm{cm}$ electrical conductivity) with or without sanitizer. The salt solution was used to improve electrical contact between electrodes and have a more homogeneous electric field distribution. The PEF treatment conditions in this study were $2 \mathrm{kV} / \mathrm{cm}$ field strength, $1 \mu \mathrm{s}$ pulse width, and 100 pulses per second. The treatment times were 2,4 , and 6 min and treatment temperatures were $22^{\circ} \mathrm{C}$. Approximately 4,6 , and $8^{\circ} \mathrm{C}$ temperature increase was observed for 2,4 , and 6 min treatment time, respectively. These PEF parameters were selected based on our previous trials considering the PEF process system power limit, operation stability, and microbial inactivation. 
After PEF treatment, the blueberry samples were taken out from the chamber, blotted with paper towels to remove excess water, weighed, and subject to microbial and quality analyses.

\subsection{Microbiological analysis}

Untreated and treated blueberry samples were transferred into individual sterile stomacher bags and then stomached in $10 \mathrm{~mL}$ of $0.1 \%$ peptone water for $1 \mathrm{~min}$. Serial dilutions of the resultant suspensions were made in $0.1 \%$ peptone water and surface-plated $(100 \mu 1 /$ plate $)$ onto PALCAM agar plates (BBL/Difco, Sparks, MD) with PALCAM selective supplement (Oxoid, England) for Listeria, tryptic soy agar (TSA: BBL/Difco) for E. coli, Plate Count Agar (PCA, BBL/Difco) plates for aerobic mesophilic bacteria and dichloran rose bengal chloranphenicol agar (DRBC, Merck, Germany) plates for yeasts and molds (Y\&M). PALCAM, TSA, and PCA plates were incubated at $37^{\circ} \mathrm{C}$ up to $48 \mathrm{~h}$, while DRBC plates were incubated at $25^{\circ} \mathrm{C}$ for 4 to 5 days. Colony forming units (CFU) were then enumerated.

\subsection{Color and Texture analysis}

Color analysis was conducted at $25 \pm 2^{\circ} \mathrm{C}$ using a portable General Colorimeter (JZ-300, Kingwell Instrument Col. Ltd., Guangdong, China) in the reflectance mode. Color was expressed in $L^{*}, a^{*}$ and $b^{*}$ values. Color analysis was conducted using ten blueberries for each pretreatment and the results were averaged.

Compression tests were performed to evaluate any changes in the hardness of blueberry samples. The hardness value $(\mathrm{g})$ is the peak force that occurs during the first compression. The force required to compress each blueberry fruit was measured individually using a TA-XT2i texture analyzer (Texture Technologies, Scarsdale, NY, USA) with a 3mm diameter stainless 
cylinder probe TA-212. The probe height was calibrated to $20 \mathrm{~mm}$ above the TA-90 base platform so that the blueberry could be aligned directly under the probe. The test settings were as followed: pretest speed of $2 \mathrm{~mm} / \mathrm{s}$ and test speeds of $2 \mathrm{~mm} / \mathrm{s}$ with an automatic trigger set to 5 grams of force; and test distance of $3 \mathrm{~mm}$ into the blueberries. Each time a set of fruits was measured, the results were the average of 10 berries.

\subsection{Determination of total phenolics and anthocyanins}

Blueberry samples $(20 \mathrm{~g})$ were homogenized and mixed with $30 \mathrm{~mL}$ of ethanol. After the mixture was centrifuged at $4000 \mathrm{x}$ g for $5 \mathrm{~min}$, blueberry residue was extracted with $85 \%$ (v/v) ethanol two times, and supernatant (total $100 \mathrm{~mL}$ ) was used to determine total phenolics content and total anthocyanin content.

Total phenolics contents in the supernatant were determined via the Folin-Ciocalteu method as described by Yu et al. (2014). Quantification was based on the calibration curve generated with gallic acid standard solutions, and contents were expressed as Gallic Acid equivalent (GAE)/g dry matter.

Anthocyanins contents in the supernatant were determined using the $\mathrm{pH}$ differential method as described by Lee et al. (2005). The absorbance of each sample diluted with $\mathrm{pH} 1.0$ and $\mathrm{pH} 4.5$ buffers at both 520 and $700 \mathrm{~nm}$ was read versus a blank cell filled with deionized water using a spectrophotometer (Genesys 10 UV, Thermo Electron Corporation, Pennsylvania, USA). The results were expressed as cyanidin-3-glucoside (C3G) equivalents/g dry matter.

To determine the compounds in peels or juice separately, blueberry samples were homogenized and filtered using double-layer cheesecloth. The solid and liquid portions of the berry homogenate were analyzed following the previously-described procedures. 


\subsection{Statistical analysis}

Ten blueberries were sampled in each experimental trial and experiments were conducted in triplicate for each treatment. The microbial counts for each replicate were transformed to log CFU/g before data analysis. Analysis of variance (ANOVA) was performed with SAS version 9.1 (SAS Institute, Inc., Cary, NC). Mean comparisons were made between the control and each treatment replicate using LSD to determine significance. Significant differences were defined at $\mathrm{P}<0.05$.

\section{Results}

\subsection{Inactivation of Microorganisms}

The first trail was conducted to investigate the efficacy of the PEF treatment conditions in microbial inactivation for blueberries in salt water without addition of sanitizer (T100). Figure 2 shows that approximately $1 \log \mathrm{CFU} / \mathrm{g}$ of artificially inoculated $E$. coli on blueberries was reduced due to "washing" effect by soaking samples in salt solution (CK1). The PEF treatment achieved additional $0.5 \log$ reduction after 4 and $6 \mathrm{~min}$. There were no significant ( $>>0.05)$ difference in microbial inactivation between 4 and 6 min treatments. Therefore, the treatment time of 6 min was excluded for further studies with sanitizing solutions.

Figure 3 shows the effect of sanitizer concentrations on the survival of artificially inoculated E. coli on blueberries after PEF treatments. The initial E. coli cell populations were approximately $4.3 \log \mathrm{CFU} / \mathrm{g}(\mathrm{CK} 0)$. E. coli populations were reduced by 1 and $1.5 \log \mathrm{CFU} / \mathrm{g}$ on non-PEF (CK1) and PEF treated (PEF) samples in the solution without sanitizer (0\%T100), which was similar to the result in Figure 2. Addition of 0.25\% sanitizer (0.25\%T100) 
significantly increased the inactivation rate and further reduced E. coli populations by 1.8 and $2.9 \log \mathrm{CFU} / \mathrm{g}$ respectively. However, an increase in the concentration to $0.5 \%$ (0.5\%T100) did not show further reduction of E. coli populations. Similar result was observed for Listeria on blueberry samples (Figure 4). Therefore, the treatment solution with $0.25 \%$ T100 was used for further studies.

Figure 5 shows the effect of PEF treatment time on the inactivation of Listeria on blueberry samples. PEF treatment at 2 and 4 min reduced Listeria populations by 2.3 and $2.6 \log$ CFU/g, respectively. Listeria populations in blueberry samples (CK1) which were soaked in sanitizing solution and not subject to PEF treatment were reduced by less than $1 \log$ CFU/g.

Same PEF treatments were applied to natural microflora on blueberries. Figure 6 shows the effects of PEF treatment on the inactivation of native bacteria (A) and yeasts and molds (B) on blueberry samples. PEF treatment reduced total bacterial counts from $2.6 \log \mathrm{CFU} / \mathrm{g}$ to undetectable levels ( $<0.7 \log \mathrm{CFU} / \mathrm{g}$ ) after $2 \mathrm{~min}$, achieving more than $2.5 \log$ reduction (Figure 6A). Blueberries had higher initial Y\&M counts than native bacteria (viz., ca. $4.8 \log \mathrm{CFU} / \mathrm{g}$ ). After PEF treatments, populations were reduced to 3.5, and $3.1 \log$ CFU/g for 2 and $4 \mathrm{~min}$, respectively (Figure 6B).

Unlike E. coli and Listeria, native microorganisms were less sensitive to sanitizer solutions. When blueberry samples were placed in the sanitizing solution for 4 min without PEF treatment (CK1), only $0.2 \log \mathrm{CFU} / \mathrm{g}$ reduction of bacteria and $0.7 \log \mathrm{CFU} / \mathrm{g}$ reduction of Y\&M were obtained (Figure 6).

\subsection{Changes in Quality and Nutrient Concentration}


The effect of PEF on the visual appearance, surface color, compression firmness, and total anthocyanins and phenolic compounds in blueberries was determined immediately after treatments. Based on visual inspection, blueberries did not display any signs of gross physical damage, compared to the control (Figure 7). Surface color measurements (L*, a*, $b^{*}$ values) were not significantly impacted by PEF treatments (Table 1). However, a trend towards reduction in compression firmness was detected when blueberries were treated with sanitizer solution and PEF (Table 1).

Anthocyanin concentration in whole fruit increased from 101 units to 110 units after the PEF treatment, and total phenolics were increased from 111 to 152 units, which corresponded to a $10 \%$ and $23 \%$ increase in anthocyanins and total phenolics, respectively, compared to the controls (non-PEF treated) (Figure 8).

In addition, PEF treatments also affected the distribution of anthocyanins and total phenolics in blueberries. Higher anthocyanin concentration and total phenolics were observed in the juice portions, while lower concentrations were present in the peel/skin portions following PEF treatment (Figure 9). Ninety percent of anthocyanins in peel and $10 \%$ in juice changed to $85 \%$ in peels and $15 \%$ in juice after PEF treatments. Similarly, for total phenolics, $86 \%$ in peel and $14 \%$ in juice were changed to $82 \%$ in peel and $18 \%$ in juice.

\section{Discussion}

PEF is a promising alternative to current juice and beverage processing technologies. Nevertheless, PEF was not effective in reducing the numbers of microorganisms on whole fruits without the aid of sanitizing solutions, potentially, because the applied field strength we used (2 $\mathrm{kV} / \mathrm{cm}$ ) is limited in efficacy compared to the higher field strengths (viz., $25-45 \mathrm{kV} / \mathrm{cm}$ ) used 
with liquid products. To compensate for low field strength, a longer treatment time (2-6 min) was used in this study compared to $250 \mu$ s for juice pasteurization (Guo et al., 2014). In addition, a sanitizing solution was used in combination with PEF treatments. It is a general rule that a liquid medium with a specific conductivity must be used for PEF processing to provide uniform PEF treatment. Liquid food itself, or a salt water carrier for solid food, is usually used. Two advantages were observed when the sanitizer solution was used in combination with PEF. One advantage is that the combination increased the inactivation rates of E. coli, Listeria, and native microorganisms on blueberries. When salt water was used alone, the PEF treatment only achieved less than $0.5 \log \mathrm{CFU} / \mathrm{g}$ reduction of $E$. coli populations (Figure 2). The combination was able to achieve up to a 2.9 and $3 \log$ CFU/g reduction of E. coli (Figure 3) and Listeria (Figure 4 and 5), as well as $2 \log$ CFU/g reduction of native microorganisms (Figure 6). Use of the sanitizing solution alone caused a very limited reduction in bacterial populations. Davidson et al. (2013) reduced E. coli O157:H7 populations, which were artificially inoculated onto iceberg lettuce by $0.93 \log \mathrm{CFU} / \mathrm{g}$ when washed for 90 s with $50 \mathrm{ppm}$ of peroxyacetic acid (Tsunami 100), which was not significantly $(\mathrm{P}<0.05)$ more effective than water alone. It has been reported that PEF treatments with different treatment conditions cause death or injury of microbial cells and the sub-lethally injured cells could recover depending on growth environment (Jin et al., 2014; Gurtler et al., 2010). Sanitizing solution or other antimicrobials increases destruction of the microbial cytoplasmic membrane as well as preventing cell repair of survivors from PEF treatments (Nguyen \& Mittal, 2007). Therefore, the combination in the present study can enhance their killing effect on microorganisms, particularly on those injured during PEF processing. The other advantage is that the combination treatment inactivated microorganisms in the solution and reduced the risk of cross-contamination, if the solution is re-circulated. Our test 
demonstrates that there were no detectable cells in the carrier solution used for all treatments (data not shown).

The softening of PEF treated blueberries may be due to cell membrane breakdown by PEF treatment, known as electroporation. A number of publications have reported changes in plant tissue integrity after PEF processing that resulted in texture softening (Asavasanti et al., 2010; De Vito et al., 2008; Gonzalez et al., 2010a, 2010b; Lebovka et al., 2004). From a sensory standpoint, maximum force represents the hardness of the food sample (Bourne, 2002). Decreases in hardness may negatively affect sensory quality of whole fresh fruit that requires a firm texture (or may impute a positive effect for those fruits in which soft texture is preferred). Therefore, the results from the present study suggest that PEF processing is more suitable for fruits that require softening before consumption.

Anthocyanins reside in vacuoles, mostly found on the skin of blueberries (Bjoroy et al., 2009). Total anthocyanins demonstrated a significant increase in C3G equivalents after PEF treatment (Figure 8). This result indicates that in this particular range of processing conditions, the PEF treatment may modify the mechanism of anthocyanins degradation by affecting the molecules involved in the kinetics of the reaction, such as enzymes. Barba et al. (2013) and Ferrari et al. (2010) observed similar results when they studied blueberry and pomegranate juice properties after high pressure treatments. Similarly, phenols were significantly increased (23\%), after PEF treatment. This increase in total phenolic content may be related to increased extractability of some of the antioxidant components following PEF processing. Other studies have reported an increase in total phenolic content of blueberry juice (Barba et al., 2013) and grape by-products (Corrales et al., 2008), following high pressure processing treatment. In 
contrast, some other non-thermal interventions, such as cold plasma (Lacombe et al., 2015) and ozone (Tiwari et al., 2009), significantly reduced anthocyanins in fruits or juices.

PEF treatment increased the concentrations of anthocyanins and total phenolics and changed their distribution throughout the blueberries. Higher concentrations were obtained in juices than in peels after PEF treatment (Figure 9). It can be explained that PEF treatment increases the permeabilization of cell membranes due to the electroporation phenomenon (Kotnik et al., 2012). Studies on extraction of intracellular compounds from plant tissues treated with PEF have shown that a higher yield of active compounds was obtained after PEF treatment (Jemai \& Vorobiev, 2006; Praporscic et al., 2007; Schilling et al., 2007; Turk et al., 2012). PEF treatment has recently shown good results for the enhancement of polyphenols and anthocyanins extraction from grape wastes (Boussetta et al., 2012, 2009; Brianceau et al., 2015; Corrales et al., 2008). Increases of those nutrients in the juice portion after PEF treatment would benefit juice processing by allowing for the production of juice with better nutritional properties.

Blueberries can be consumed as fresh fruit, and also can be used to make juice, jam, or dehydrated products. PEF treatment significantly reduced initial microbial loads and improved the nutritional values of fresh whole fruits, which is an important step in producing high quality and safe products derived from berries. The FDA requires that juice pasteurization reduce target pathogens by $5 \log$ CFU. This mandatory requirement is challenging for most nonthermal processes without scarifying the quality and nutritional properties of juice products. However, multiple (i.e., hurdle) treatments are permitted to meet this requirement, as long as juices are processed in the same facility (FDA, 2004), hence, two-stage processing is possible. In the first stage, PEF pretreatment could be applied to whole fruits to achieve a ca. 2 log reduction, allowing for the production of juice with high nutritional value. In the second stage, the juice 
would be subject to further treatment, such as PEF, at high intensity and short time or by other nonthermal processing treatments, to achieve an additional 3 log reduction. In this way, less severe processing conditions could be applied for each treatment, not only allowing for the preservation or improvement of quality and nutritional properties, but also meeting the FDA 5$\log$ reduction requirement for juice pasteurization. Similarly, PEF has the potential for flexible applications in other derived fruit products.

To our best knowledge, this is the first study that investigates the use of a sanitizing solution as a PEF treatment medium and PEF-induced inactivation of potential pathogenic and spoilage microorganisms on blueberries, while considering its effect on their quality and nutrition. Future studies will address the feasibility for scale-up of this technology to pilot plant as well as its application in derived products. Optimal PEF parameters (field strength, pulse width, frequency, total treatment time), and other parameters (treatment temperature, type of treatment liquid media, etc.) will be further evaluated.

\section{Conclusion}

The capability of PEF to be utilized as a postharvest sanitization process was demonstrated in this study by the logarithmic reduction of E. coli, Listeria and native microorganisms. This combination of PEF plus a sanitizer solution will potentially allows for efficient decontamination of blueberries and other small fruits. The significance of PEF pretreatment in inactivation of microorganisms on whole fruits is to make the products safer at the beginning of processing; thus, producers have more flexibility to make pathogen-free products, such as fresh-like whole fruits, or their derived products such as purees, pastes, or juices with reduced populations of pathogenic or spoilage contaminants. In addition, PEF 
processing preserved the berries visual appearance and improved their nutritional properties, while softening their texture, suggesting the usefulness of further research for various fruits and their derived products.

\section{Acknowledgments}

The authors wish to thank Anita Parameswaran and Kimberly Sokorai for their excellent technical assistance. Author Yuanshan Yu wishes to thank the Program of Innovative Talents Training Abroad from Guangdong Academy of Agricultural Sciences (China) for financial support to work at ERRC-ARS-USDA. All work was done at ERRC-ARS-USDA.

\section{References}

Alexandre, E.M.C., Brandão, T.R.S., \& Silva, C.L.M. (2012). Assessment of the impact of hydrogen peroxide solutions on microbial loads and quality factors of red bell peppers, strawberries and watercress. Food Control, 27(2), 362-368.

Alvaro, J.E., Moreno, S., Dianez, F., Santos, M., Carrasco, G., \& Urrestarazu, M. (2009). Effects of peracetic acid disinfectant on the postharvest of some fresh vegetables. Journal of Food Engineering, 95, 11-15.

Angersbach, A., Heinz, V., \& Knorr, D. (2000). Effects of pulsed electric fields on cell membranes in real food systems. Innovative Food Science and Emerging Technologies, 1, 135-149.

Asavasanti, S., Ersus, S., Ristenpart, W., Stroeve, P., \& Barrett, D.M. (2010). Critical electric field strengths of onion tissues treated by pulsed electric fields. Journal of Food Science, 75, E433-E443. 
Barba, F.J., Esteve, M.J., \& Frigola, A. (2013). Physicochemical and nutritional characteristics of blueberry juice after high pressure processing. Food Research International, 50, 545549.

Bazhal, M.I., Lebovka, N.I., \& Vorobiev, E. (2001). Pulsed electric field treatment of apple tissue during compression for juice extraction. Journal of Food Engineering, 50, 129-139.

Bjoroy, O., Rayyan, S., Fossen, T., \& Andersen, O.M. (2009). Structural properties of anthocyanins: Rearrangement of C-glycosyl-3-deoxyanthocyanidins in acidic aqueous solutions. Journal of Agricultural and Food Chemistry, 57, 6668-6677.

Boussetta, N., Lebovka, N., Vorobiev, E., Adenier, H., Bedel-Cloutour, C., \& Lanoisellé, J.L. (2009). Electrically assisted extraction of soluble matter from chardonnay grape skins for polyphenol recovery. Journal of Agricultural and Food Chemistry, 57, 1491-1497.

Boussetta, N., Vorobiev, E., Le, L.H., Cordin-Falcimaigne, A., \& Lanoisellé, J.-L. (2012). Application of electrical treatments in alcoholic solvent for polyphenols extraction from grape seeds. LWT - Food Science and Technology, 46, 127-134.

Bourne, M. (2002). Food texture and viscosity: concept and measurement. 2nd ed. Calif.: Academic Press. 416 p.

Brianceau, S., Turk, M., Vitrac, X., \& Vorobiev, E. (2015). Combined densification and pulsed electric field treatment for selective polyphenols recovery from fermented grape pomace. Innovative Food Science and Emerging Technologies, 29, 2-8.

Calder, L., Simmons, G., Thornley, C., Taylor, P., Pritchard, K., Greening, G., \& Bishop, J. (2003). An outbreak of hepatitis A associated with consumption of raw blueberries. Epidemiology and Infection, 131, 745-751. 
CDC (Center for Disease Control and Prevention). (2015). Foodborne Outbreak Online Database. http://wwwn.cdc.gov/foodborneoutbreaks/. Accessed May 2, 2016.

Corrales, M., Toepfl, S., Butz, P., Knorr, D., \& Tauscher, B. (2008). Extraction of anthocyanins from grape by-products assisted by ultrasonics, high hydrostatic pressure or pulsed electric fields: A comparison. Innovative Food Science and Emerging Technologies, 9, 85-91.

Crowe, K.M., Bushway, A.A., \& Bushway, R.J. (2005). Effects of alternative postharvest treatments on the microbiological quality of lowbush blueberries. Small Fruits Review, 4, 29-39.

Davidson, G.R., Buchholz, A.L., \& Ryser, E.T. (2013). Efficacy of commercial produce sanitizers against nontoxigenic Escherichia coli O157:H7 during processing of Iceberg lettuce in a pilot-scale leafy green processing line. Journal of Food Protection, 76, 18381845.

De Vito, F., Ferrari, G., Lebovka, N.I., Shynkaryk, N.V., \& Vorobiev, E. (2008). Pulse duration and efficiency of soft cellular tissue disintegration by pulsed electric fields. Food and Bioprocess Technology, 1, 307-313.

EPA (1998). Environmental Protection Agency. Rules and Regulations. National Primary Water Regulation: Disinfectants and Disinfection by Products, vol. 63, no. 241.

FDA. (2004). Guidance for Industry: Juice HACCP Hazards and Controls Guidance First Edition; Final Guidance. http://www/fda/gov/Food/GuidanceRegulation/GuidanceDocumentsRegulatoryInformation/ Juice/ucm072557.htm. Accessed May 4, 2016 
Ferrari, G., Maresca, P., \& Ciccarone, R. (2010). The application of high hydrostatic pressure for the stabilization of functional foods: Pomegranate juice. Journal of Food Engineering, $100,245-253$.

Gonzalez, M.E., Anthon, G.E., \& Barrett, D.M. (2010a). Onion cells after high pressure and thermal processing: Comparison of membrane integrity changes using different analytical methods and impact on tissue texture. Journal of Food Science, 75, E426-E432.

Gonzalez M.E., Jernstedt J.A., Slaughter D.C., \& Barrett D.M. (2010b). Influence of cell integrity on textural properties of raw, high pressure, and thermally processed onions. Journal of Food Science, 75, E409-E416.

Guo, M., Jin, T.Z., Geveke, D.J., Fan, X., Sites, J.E., \& Wang, L. (2014). Evaluation of microbial stability, bioactive compounds, physicochemical properties, and consumer acceptance of pomegranate juice processed in a commercial scale pulsed electric field system. Food and Bioprocess Technology, 7, 2112-2120.

Gurtler, J.B., Bailey, R.B., Geveke, D.J., \& Zhang, H.Q. (2011). Pulsed electric field inactivation of $E$. coli $\mathrm{O} 157: \mathrm{H} 7$ and non-pathogenic surrogate $E$. coli in strawberry juice as influenced by sodium benzoate, potassium sorbate, and citric acid. Food Control, 22, 16891694.

Gurtler, J.B., Rivera, R.B., Zhang, H.Q., \& Geveke, D.J. (2010). Selection of surrogate bacteria in place of E. coli $\mathrm{O} 157: \mathrm{H} 7$ and Salmonella typhimurium for pulsed electric field treatment of orange juice. International Journal of Food Microbiology, 139, 1-8.

Hung, Y.-C., Tilly, P., \& Kim, C. (2010). Efficacy of electrolyzed oxidizing (EO) water and chlorinated water for inactivation of Escherichia coli $\mathrm{O} 157: \mathrm{H} 7$ on strawberries and broccoli. Journal of Food Quality, 33, 559-577. 
Jemai, A.B., \& Vorobiev, E. (2006). Pulsed electric field assisted pressing of sugar beet slices: Towards a novel process of cold juice extraction. Biosystems Engineering, 93, 57-68.

Jin, T.Z., Guo, M., \& Zhang, H.Q. (2015). Upscaling from benchtop processing to industrial scale production: More factors to be considered for pulsed electric field food processing. Journal of Food Engineering , 146, 72-80.

Jin, T., Guo, M., \& Yang, R. (2014). Combination of pulsed electric field processing and antimicrobial bottle for extending microbiological shelf-life of pomegranate juice. Innovative Food Science and Emerging Technologies, 26, 153-158.

Jin, T., Zhang, H., Hermawan, N., \& Dantzer, W. (2009a). Effect of pH and temperature on inactivation of Salmonella typhimurium DT104 in liquid whole egg by pulsed electric fields. International Journal of Food Science and Technology, 44, 367-372.

Jin, Z.T., Zhang, H.Q., Li, S.Q., Kim, M., Dunne, C.P., Yang, T., Wright, A.O., \& Venter- Gains, J. (2009b). Quality of applesauces processed by pulsed electric fields and HTST pasteurization. International Journal of Food Science and Technology, 44, 829-839.

Jin, Z.T., \& Zhang, Q.H. (1999). Pulsed electric field inactivation of microorganisms and preservation of quality of cranberry juice. Journal of Food Processing and Preservation, $23,481-497$.

Kim, C., \& Hung, Y.-C. (2012). Inactivation of E. coli O157:H7 on blueberries by electrolyzed water, ultraviolet light, and ozone. Journal of Food Science, 77, M206-M211.

Kotnik, T., Kramar, P., Pucihar, G., Miklavcic, D., \& Tarek, M. (2012). Cell membrane electroporation - Part 1: The phenomenon. IEEE Electrical Insulation Magazine, 28, 14 23. 
Lacombe, A., Niemira, B.A., Gurtler, J.B., Fan, X., Sites, J., Boyd, G., \& Chen, H. (2015). Atmospheric cold plasma inactivation of aerobic microorganisms on blueberries and effects on quality attributes. Food Microbiology, 46, 479-484.

Lebovka, N.I., Praporscic, I., \& Vorobiev, E. (2004). Combined treatment of apples by pulsed electric fields and by heating at moderate temperature. Journal of Food Engineering, 65, 211-217.

Lee, J.M., Durst, R.W., \& Wrolstad, R.E. (2005). Determination of total monomeric anthocyanins pigment content of fruit juices, beverages, natural colorants, and wines by the pH differential method: Collaborative study. Journal of AOAC International, 88, 1269-1278.

Luna, R.E., Mody, R., \& Griffin, P.M. (2010). Memorandum: Non-O157 Shiga toxin producing E. coli (STEC) outbreaks, United States. http://blogs.cdc.gov/publichealthmatters/files/2010/05/nono157stec_obs_052110.pdf. Accessed May 4, 2016.

Miller, B.D., Rigdon, C.E., Robinson, T.J., Hedberg, C., \& Smith, K.E. (2013). Use of global trade item numbers in the investigation of a Salmonella newport outbreak associated with blueberries in Minnesota, 2010. Journal of Food Protection, 76, 762-769.

Min, S., Jin, Z.T., Min, S.K., Yeom, H., \& Zhang, Q.H. (2003). Commercial-Scale pulsed electric field processing of orange juice. Journal of Food Science, 68, 1265-1271.

Nguyen, P., \& Mittal, G.S. (2007). Inactivation of naturally occurring microorganisms in tomato juice using pulsed electric field (PEF) with and without antimicrobials. Chemical Engineering and Processing, 46 (4), 360-365. 
Praporscic, I., Shynkaryk, M.V., Lebovka, N.I., \& Vorobiev, E. (2007). Analysis of juice colour and dry matter content during pulsed electric field enhanced expression of soft plant tissues. Journal of Food Engineering, 79, 662-670.

Reina, L.D., Jin, Z., Zhang, H., \& Youself, A. (1998). Inactivation of Listeria monocytogenes in Milk by Pulsed Electric Fields. Journal of Food Protection, 61, 1203-1206.

Sarvikivi, E., Roivainen, M., Maunula, L., Niskanen, T., Korhonen, T., Lappalainen, M., \& Kuusi, M. (2012). Multiple norovirus outbreaks linked to imported frozen raspberries. Epidemiology and Infection, 140, 260-267.

Schilling, S., Alber, T., Toepfl, S., Neidhart, S., Knorr, D., Schieber, A., \& Carle, R. (2007). Effects of pulsed electric field treatment of apple mash on juice yield and quality attributes of apple juices. Innovative Food Science and Emerging Technologies, 8, 127-134.

Tiwari, B.K., O'Donnell, C.P., \& Cullen, P.J. (2009). Effect of non thermal processing technologies on the anthocyanin content of fruit juices. Trends in Food Science and Technology, 20, 137-145.

Turk, M.F., Vorobiev, E., \& Baron, A. (2012). Improving apple juice expression and quality by pulsed electric field on an industrial scale. LWT - Food Science and Technology, 49, 245250.

Whfoods (World's Healthiest Foods). (2016). Blueberries. http://www.whfoods.com/genpage.php?tname=foodspice \&dbid=8. Accessed 5-3-2016.

Wu, V.C.H., \& Kim, B. (2007). Effect of a simple chlorine dioxide method for controlling five foodborne pathogens, yeasts and molds on blueberries. Food Microbiology, 24, 794-800. 
Yu, Y.S., Xu, Y.J., Wu, J.J., Xiao, G.S., Fu, M.Q., \& Zhang, Y.S. (2014). Effect of ultra-high pressure homogenization processing on phenolic compounds, antioxidant capacity and anti glucosidase of mulberry juice. Food Chemistry, 153, 114-120. 


\section{Figure Legends}

Figure 1. Bench-scale PEF processing system (OSU-4H Model). A: Pulse generator with controllers and oscilloscopes; B: Parallel-electrode (stainless steel) PEF treatment chamber.

Figure 2. Effects of treatment time on survival of E. coli on blueberries in salt solution without sanitizer. CK0: samples without PEF treatment and not in salt solution; CK1: samples in salt solution without PEF treatment; PEF: samples in salt solution and PEF treated. Error bars represent the standard deviation of the means.

Figure 3. Effects of sanitizer concentration on survival of E. coli on blueberries after PEF treatments. CK0: samples in sanitizing solution without PEF treatment; CK1: samples in sanitizing solution without PEF treatment for $4 \mathrm{~min}$; PEF: samples in sanitizing solution and PEF treated for 4 min. Error bars represent the standard deviation of the means.

Figure 4. Effects of sanitizer concentration on survival of L. innocua on blueberries after PEF treatments. CK0: samples in sanitizing solution without PEF treatment; CK1: samples in sanitizing solution without PEF treatment for $4 \mathrm{~min}$; PEF: samples in sanitizing solution and PEF treated for $4 \mathrm{~min}$. Error bars represent the standard deviation of the means.

Figure 5. Effect of treatment time on survival of L. innocua on blueberries after PEF treatments. CK1: samples in sanitizing solution without PEF treatment; PEF: samples in sanitizing solution and PEF treated. Error bars represent the standard deviation of the means.

Figure 6. Effects of treatment time on survival of total aerobic bacteria (A) and Y\&M (B) on blueberries after PEF treatments. CK1: samples in sanitizing solution without PEF treatment; PEF: samples in sanitizing solution and PEF treated. Error bars represent the standard deviation of the means.

Figure 7. Appearance of blueberries after PEF treatment for 0 min (A), 2 min (B), and 4 min (C).

Figure 8. Changes in anthocyanins and total phenolics concentrations in blueberries after PEF treatment. CK1: samples in sanitizing solution without PEF treatment; PEF: samples in sanitizing solution and PEF treated for $4 \mathrm{~min}$. Unit of anthocyanins: cyanidin-3-glucoside (C3G) equivalents $\mathrm{mg} / 100 \mathrm{~g}$ wet weight; unit of total phenolics: gallic acid equivalent (GAE) mg/100g wet weight. Error bars represent the standard deviation of the means.

Figure 9. Changes in the distribution of anthocyanins and total phenolics between blueberry peel and juice after PEF treatment for $4 \mathrm{~min}$. 


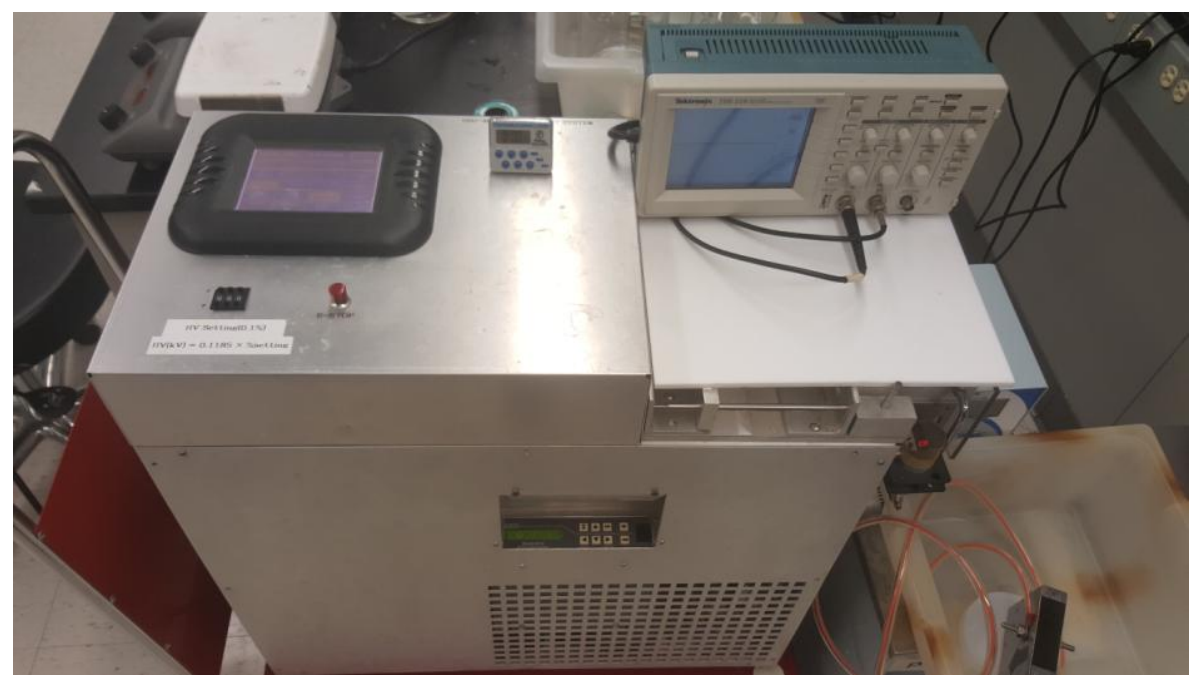

A

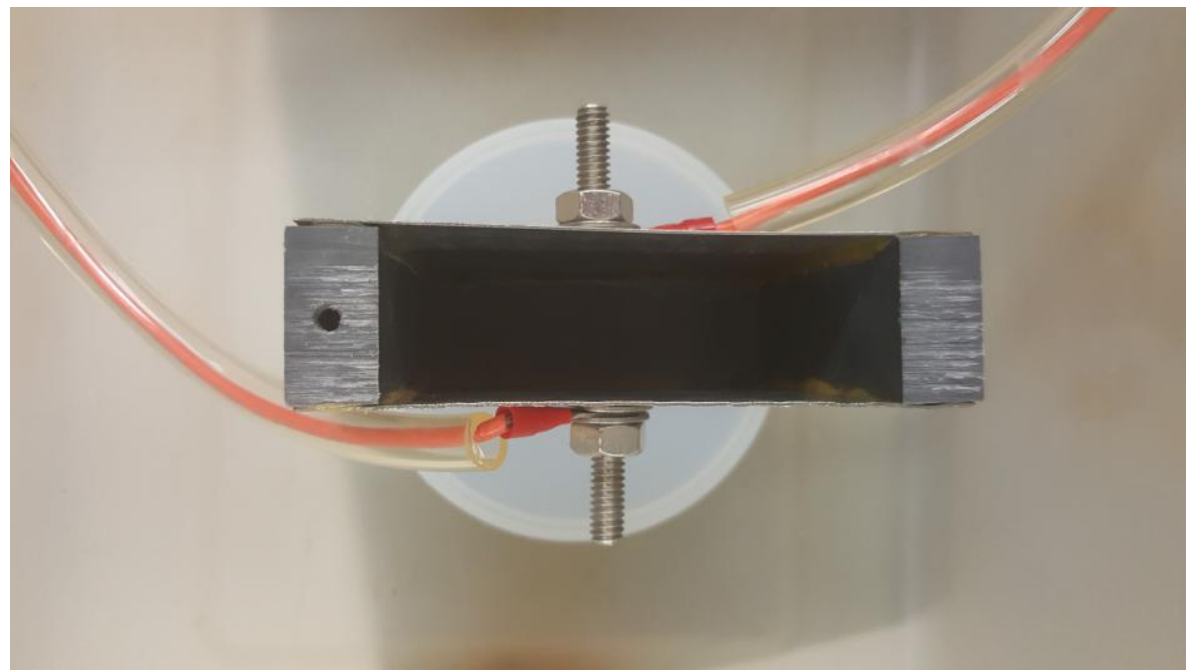

B

Figure 1. Bench-scale PEF processing system (OSU-4H Model). A: Pulse generator with controllers and oscilloscopes; B: Parallel-electrode (stainless steel) PEF treatment chamber. 


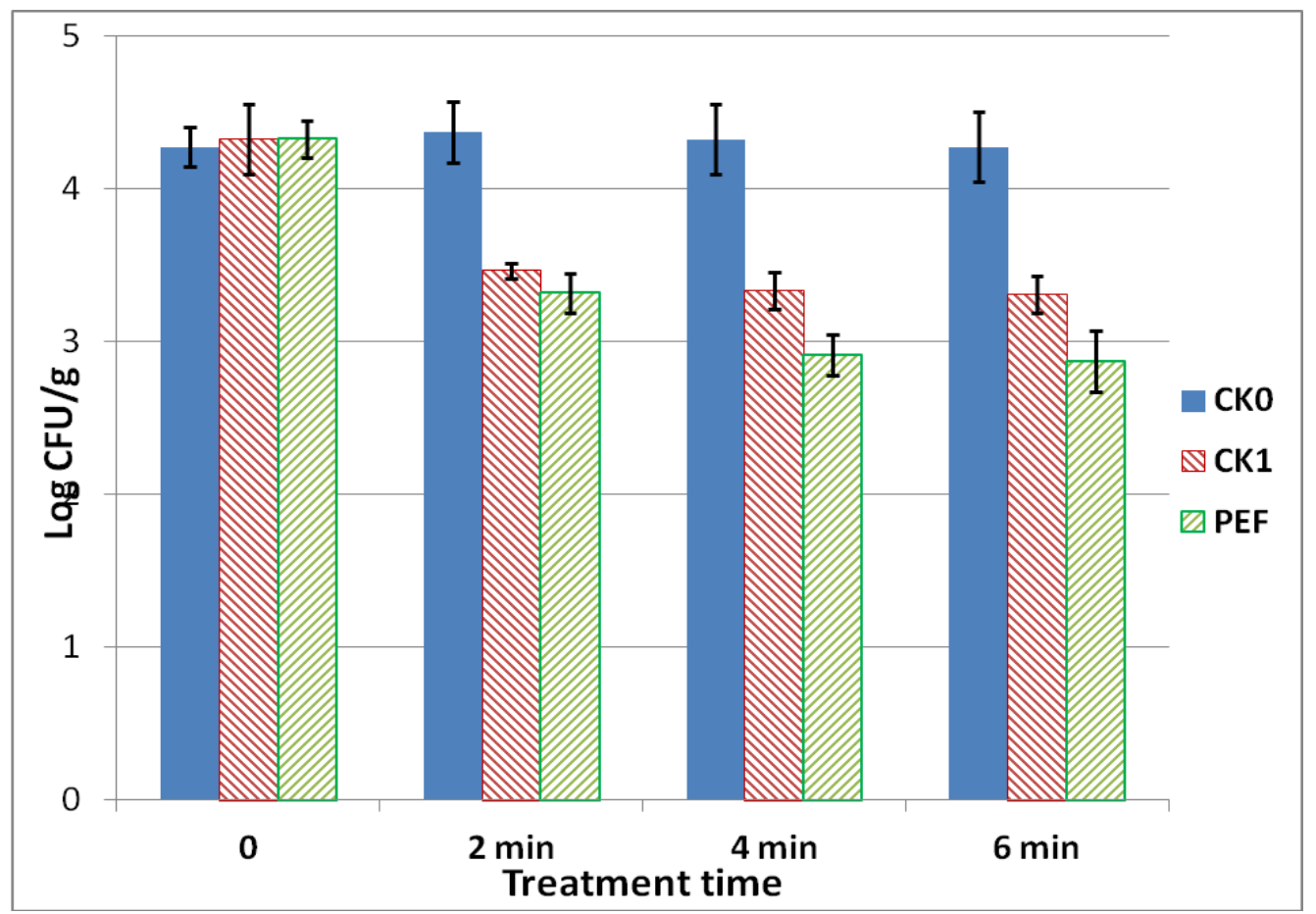

Figure 2. Effects of treatment time on survival of E. coli on blueberries in salt solution without sanitizer. CK0: samples without PEF treatment and not in salt solution; CK1: samples in salt solution without PEF treatment; PEF: samples in salt solution and PEF treated. Error bars represent the standard deviation of the means. 


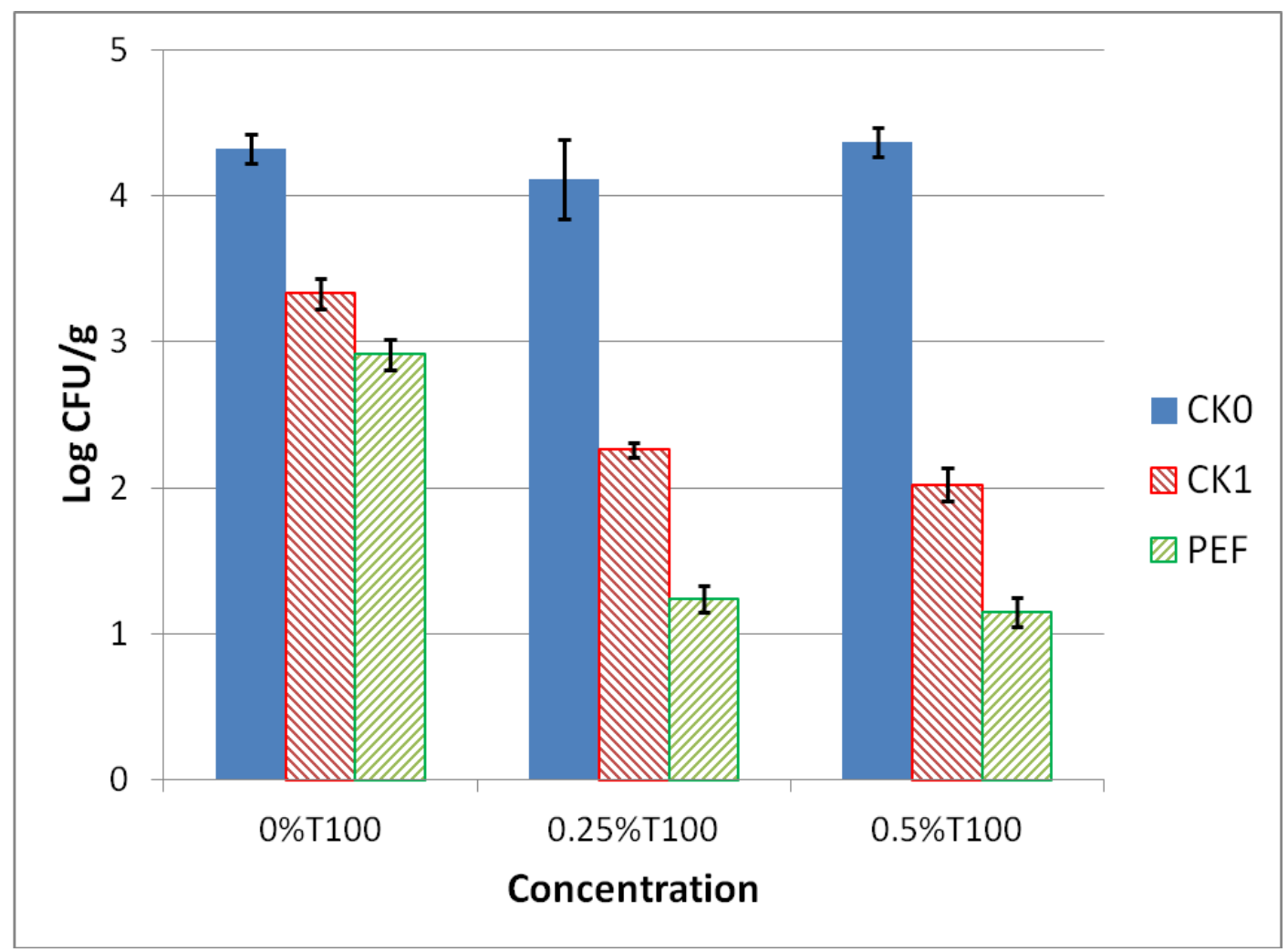

Figure 3. Effects of sanitizer concentration on survival of E. coli on blueberries after PEF treatments. CK0: samples in sanitizing solution without PEF treatment; CK1: samples in sanitizing solution without PEF treatment for $4 \mathrm{~min}$; PEF: samples in sanitizing solution and PEF treated for $4 \mathrm{~min}$. Error bars represent the standard deviation of the means. 


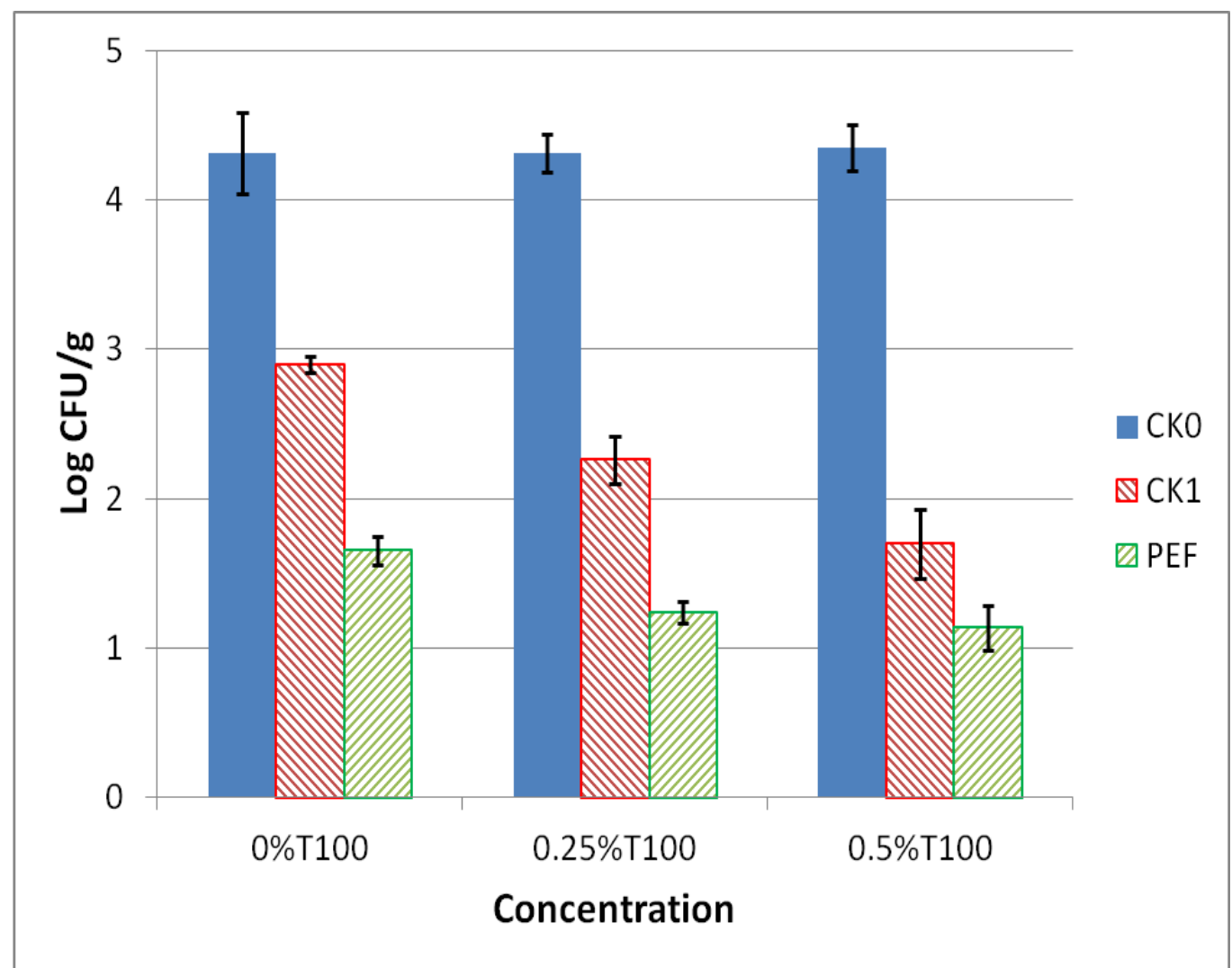

Figure 4. Effects of sanitizer concentration on survival of $L$. innocua on blueberries after PEF treatments. CK0: samples in sanitizing solution without PEF treatment; CK1: samples in sanitizing solution without PEF treatment for $4 \mathrm{~min}$; PEF: samples in sanitizing solution and PEF treated for $4 \mathrm{~min}$. Error bars represent the standard deviation of the means. 


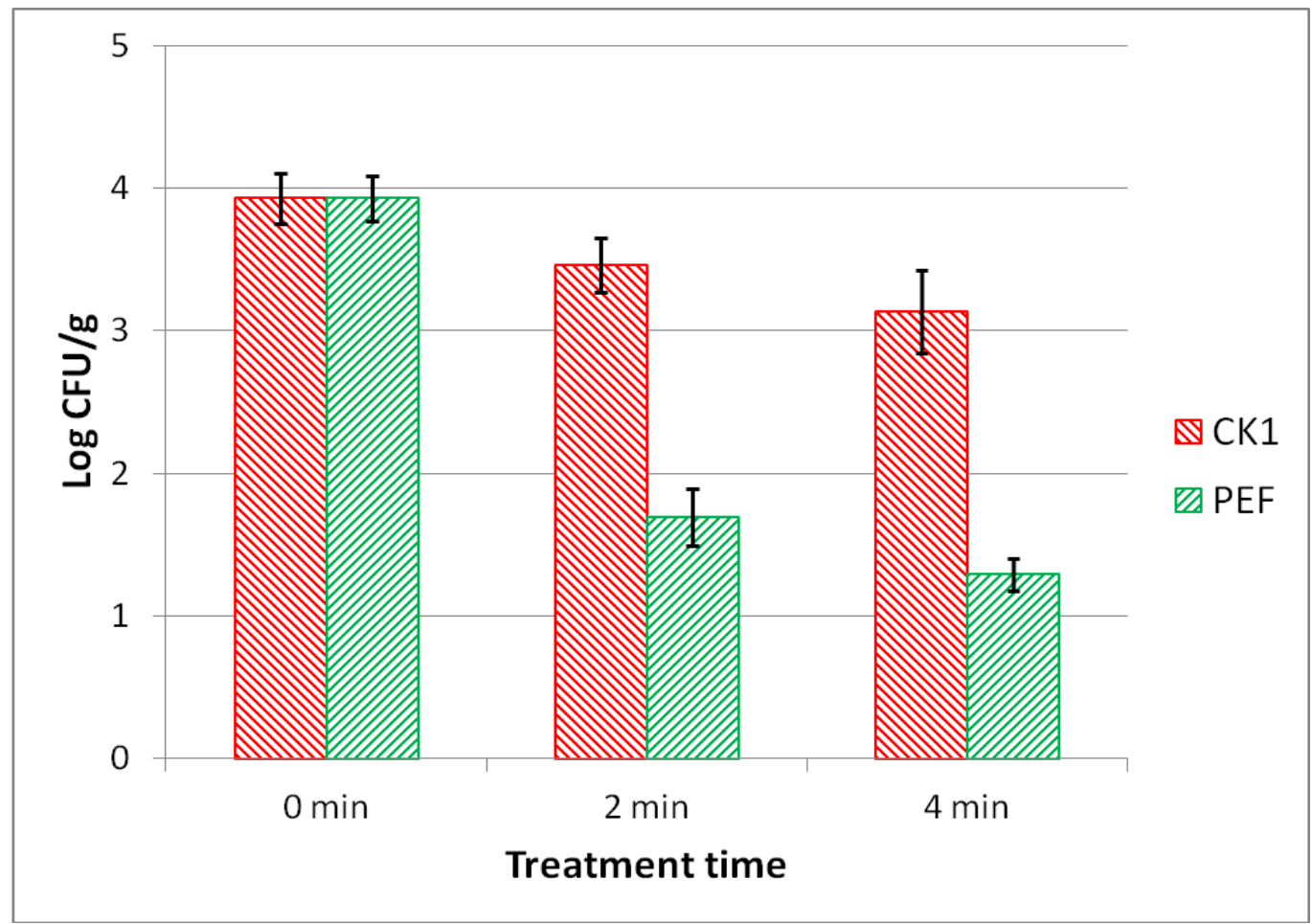

Figure 5. Effect of treatment time on survival of L. innocua on blueberries after PEF treatments. CK1: samples in sanitizing solution without PEF treatment; PEF: samples in sanitizing solution and PEF treated. Error bars represent the standard deviation of the means. 


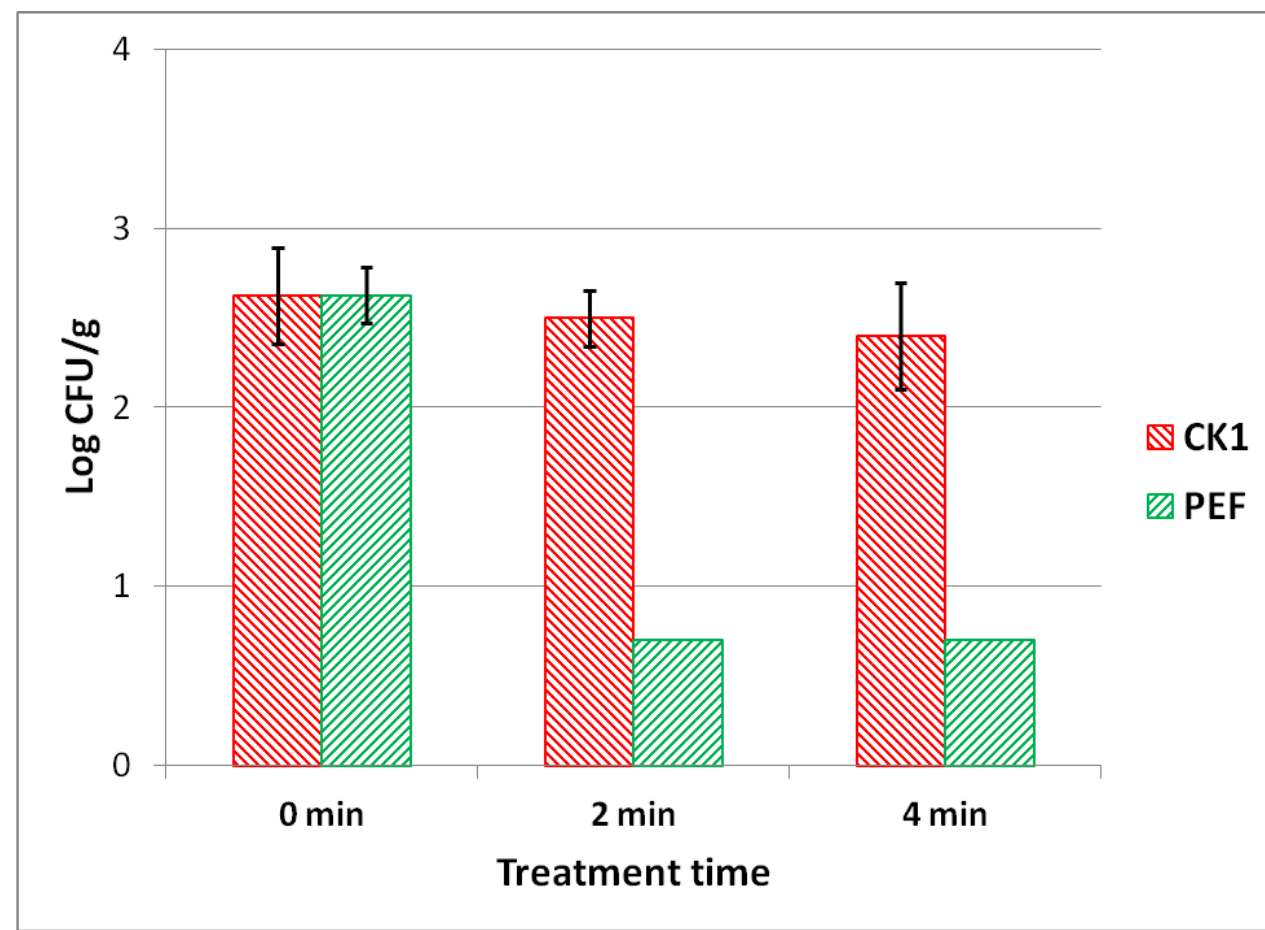

A

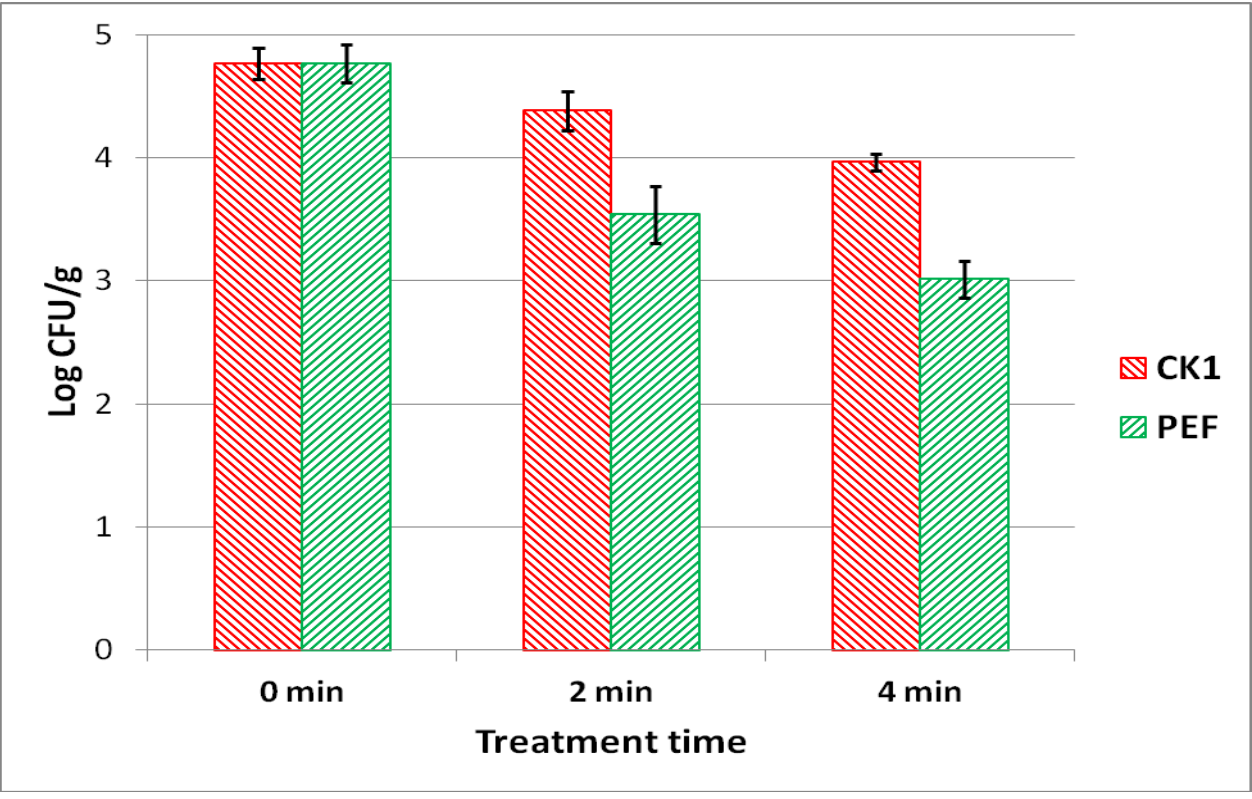

Figure 6. Effects of treatment time on survival of total aerobic bacteria (A) and Y\&M (B) on blueberries after PEF treatments. CK1: samples in sanitizing solution without PEF treatment; PEF: samples in sanitizing solution and PEF treated. Error bars represent the standard deviation of the means. 

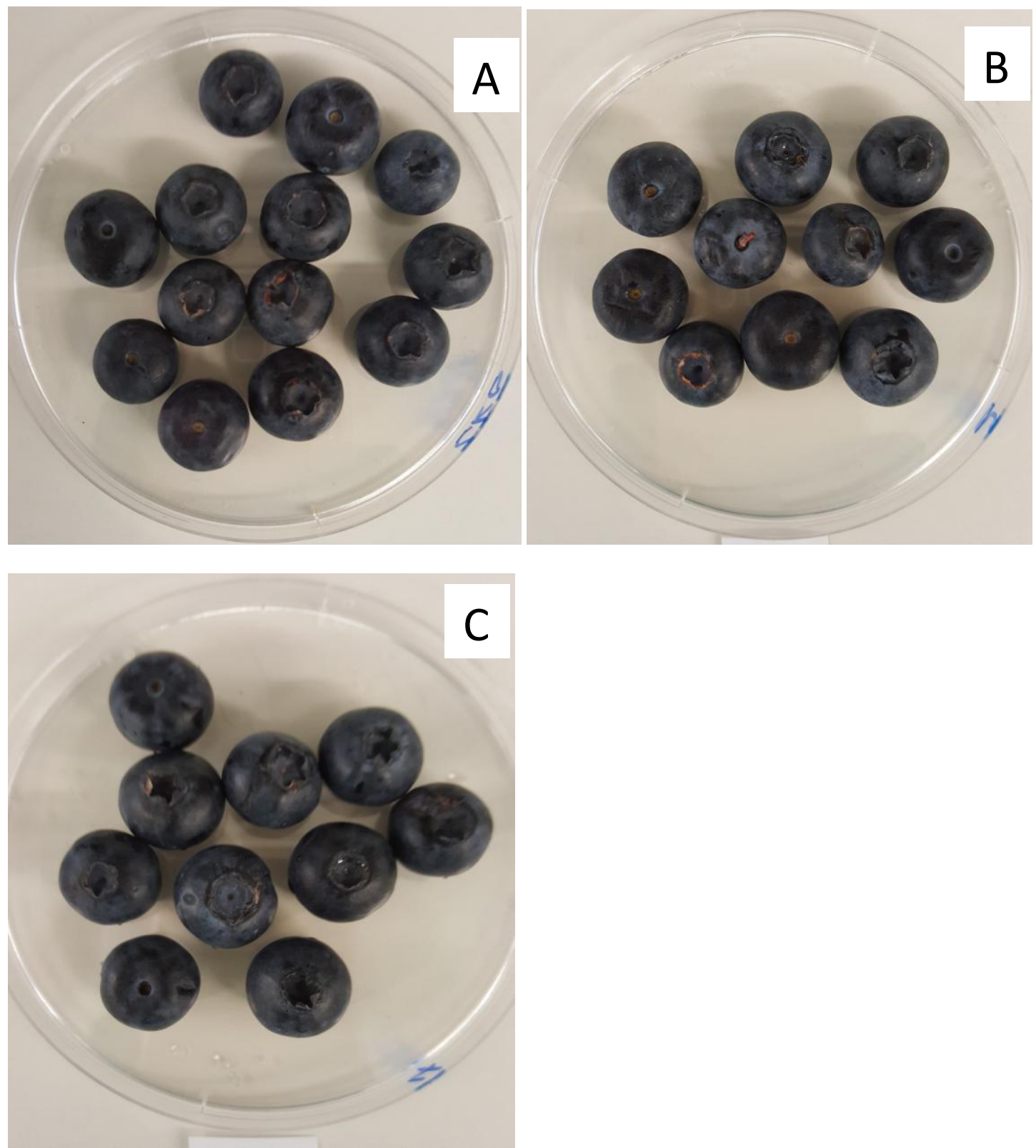

Figure 7. Appearance of blueberries after PEF treatment for 0 min (A), 2 min (B), and 4 min (C). 


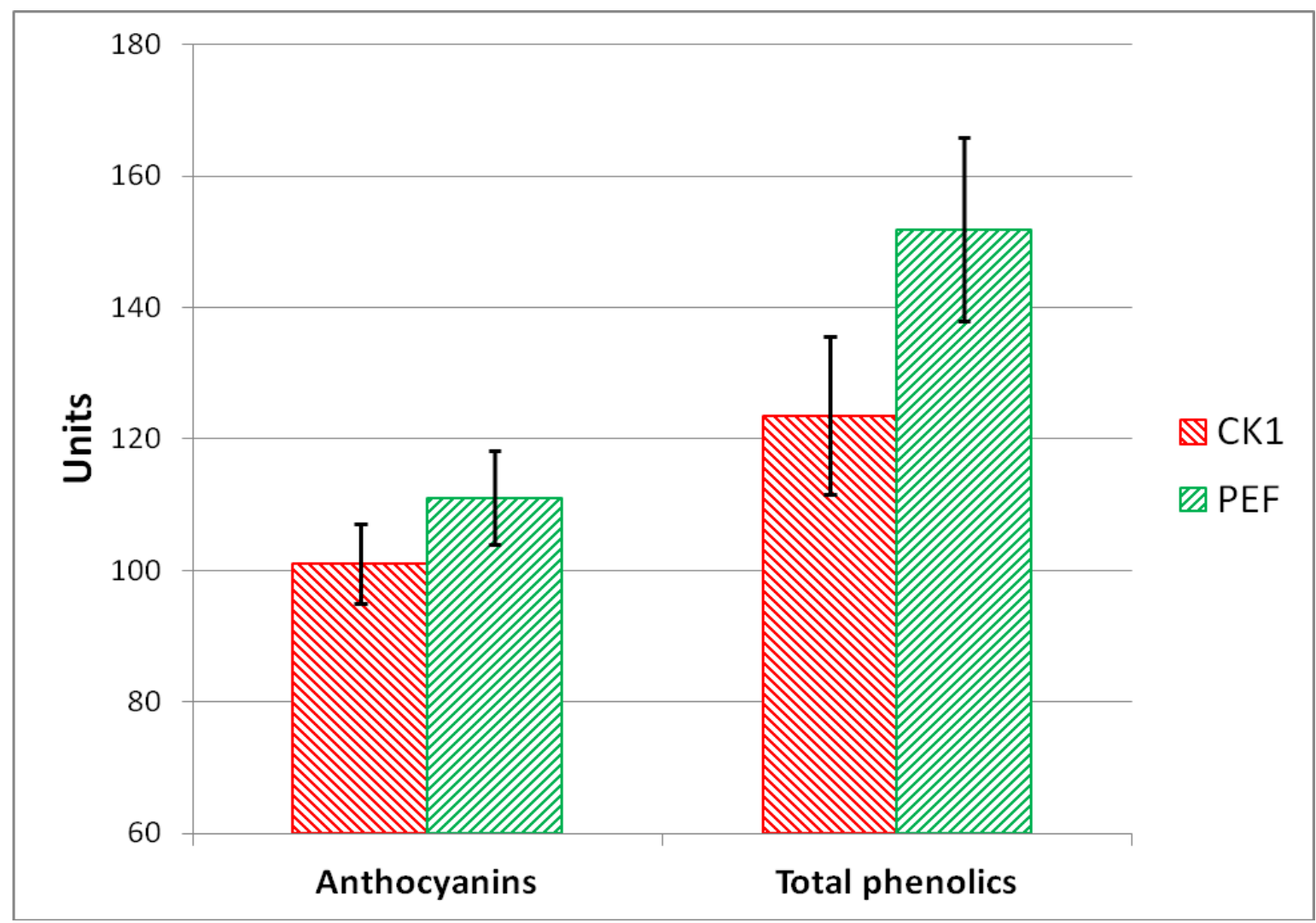

Figure 8. Changes in anthocyanins and total phenolics concentrations in blueberries after PEF treatment. CK1: samples in sanitizing solution without PEF treatment; PEF: samples in sanitizing solution and PEF treated for 4 min. Unit of anthocyanins: cyanidin-3-glucoside (C3G) equivalents $\mathrm{mg} / 100 \mathrm{~g}$ wet weight; unit of total phenolics: gallic acid equivalent (GAE) $\mathrm{mg} / 100 \mathrm{~g}$ wet weight. Error bars represent the standard deviation of the means. 


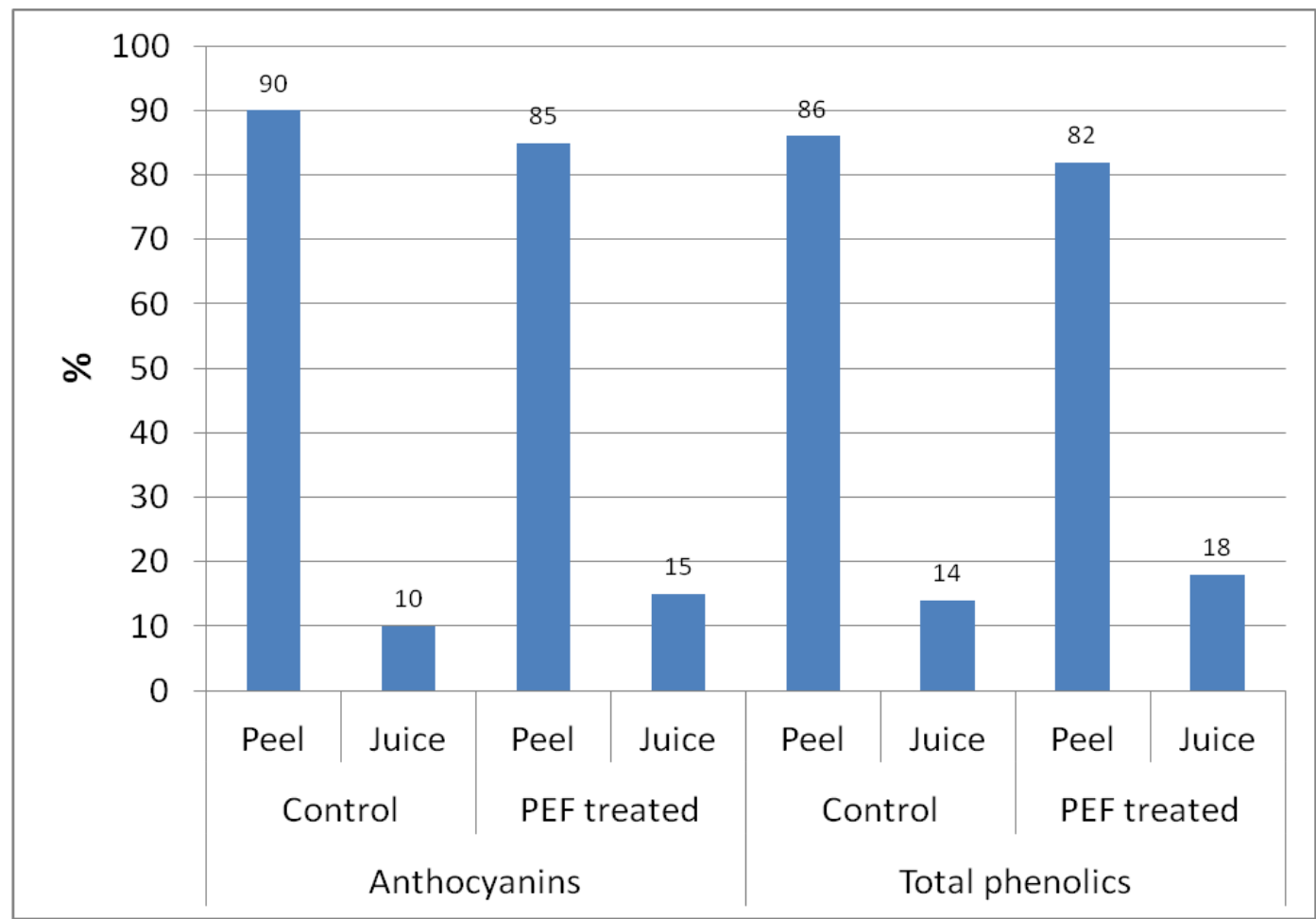

Figure 9. Changes in the distribution of anthocyanins and total phenolics between blueberry peel and juice after PEF treatment for $4 \mathrm{~min}$. 
Table 1

Color and Firmness of Blueberries after PEF treatment

\begin{tabular}{lllll}
\hline Treatment & $\mathrm{L}^{*}$ & $\mathrm{a}^{*}$ & $\mathrm{~b}^{*}$ & Hardness $(\mathrm{g})$ \\
\hline CK0 & $30.93 \pm 1.39 \mathrm{a}$ & $0.35 \pm 0.55 \mathrm{a}$ & $-3.45 \pm 0.79 \mathrm{a}$ & $499.8 \pm 134.6 \mathrm{a}$ \\
CK1 $(4 \mathrm{M})$ & $29.76 \pm 2.28 \mathrm{a}$ & $0.30 \pm 0.55 \mathrm{a}$ & $-3.06 \pm 0.95 \mathrm{a}$ & $328.9 \pm 90.8 \mathrm{~b}$ \\
PEF2M & $30.89 \pm 2.37 \mathrm{a}$ & $0.22 \pm 0.93 \mathrm{a}$ & $-3.55 \pm 1.06 \mathrm{a}$ & $143.6 \pm 44.2 \mathrm{c}$ \\
PEF4M & $30.12 \pm 1.58 \mathrm{a}$ & $0.29 \pm 0.65 \mathrm{a}$ & $-3.64 \pm 0.75 \mathrm{a}$ & $119.1 \pm 39.1 \mathrm{c}$ \\
\hline
\end{tabular}

Data are means \pm standard deviation. Means in the same column with different letters are significantly different $(\mathrm{p}<0.05)$. CK0: samples without PEF or sanitizing solution treatment; CK1(4M): samples in sanitizing solution for 4 min without PEF treatment; PEF 2M and PEF4M: samples in sanitizing solution and PEF treated for 2 or 4 min. 